\title{
Laparoscopic Common Bile Duct Exploration for Choledocholithiasis During a Pregnancy: a Case Report
}

\author{
Janis Lacis**, Ieva Rancane**, Haralds Plaudis*,**, Evita Saukane*, Guntars Pupelis*,** \\ ${ }^{*}$ Riga East Clinical University Hospital, Department of Surgery \\ $* *$ Riga Stradins University
}

\begin{abstract}
Summary
In population studies, gallstones are found in $6.5 \%$ to $8.4 \%$ of nulliparous women, and in $18.4 \%$ to $19.3 \%$ of women with two to three or more pregnancies (7). Approximately 1 in 500 to 1 in 635 women will require non-obstetrical abdominal surgery during their pregnancies. Pregnancy induced physiological hormonal changes are associated with a decrease of gallbladder motility and increased cholesterol saturation of bile, leading to biliary stone formation $(12,6,8)$. Surgical approach nowadays is the method of choice in the management of symptomatic gallstone disease during pregnancy, preferably if possible surgery should be postponed to second trimester $(7,8)$.

Preoperative radiologic imaging using magnetic resonance cholangiopancreatography is the golden standard for patients with suspected choledocholithiasis, however, its application during pregnancy is limited (9). Therapeutic endoscopic retrograde cholangiopancreatography (ERCP) may be used before, during or after laparoscopic cholecystectomy when it is indicated, but unfortunately, its application during pregnancy is associated with considerable number of complications concerning mother and fetus. Alternative approach has been developed and recommended for patients with the common bile duct (CBD) stones providing laparoscopic common bile duct exploration (LCBDE). Intraoperative cholangiography or intraoperative ultrasound (IOUS) are the methods currently used for detection of the CBD stones during laparoscopic cholecystectomy, however, IOUS can be considered as the method of choice during pregnancy (3).

IOUS is a dynamic imaging modality that provides interactive and timely information during surgical procedures. Because the transducer is in direct contact with the organ being examined, high-resolution images can be obtained that are not degraded by air, bone, or overlying soft tissue (1).
\end{abstract}

Key words: chronic cholecystitis, choledocholithiasis, LCBDE, pregnancy, intraoperative ultrasonography

\section{AIM OF THE DEMONSTRATION}

The aim of this clinical presentation is to report a clinical course of the disease of a 29-year-old patient at $22^{\text {nd }}$ week of gestation in whom one stage IOUS assisted LCBDE for choledocholithiasis was performed.

\section{CASE REPORT}

A 29-year-old Caucasian woman during week 22 of the first pregnancy was admitted to the emergency room for sudden severe midepigastric and right upper quadrant pain. Patient also described that urine had become darker. Anamnesis data revealed similar symptoms with nausea and vomiting one month ago, but patient did not look for medical assistance, because thought it was due to her pregnancy. During a physical examination abdominal muscle resistance and positive Ortner's and Murphy's signs were found, light scleral icterus was observed. The base of the uterus was at the level of umbilicus.

Laboratory values were as follows: conjugated bilirubin $13.8 \mu \mathrm{mol} / \mathrm{L}$ and total bilirubin $20.6 \mu \mathrm{mol} / \mathrm{L}, \mathrm{C}$-reactive protein (CRP) $11.6 \mathrm{mg} / \mathrm{L}$, alanine aminotransferase 64 U/L. An abdominal ultrasound examination showed normal sized gallbladder with thin walls and multiple small stones inside, slight dilatation of the CBD $(0.93$ $\mathrm{cm})$ was observed. After 24 hours of conservative therapy total serum bilirubin increased to $39.07 \mu \mathrm{mol} / \mathrm{L}$ and CRP remained $10.82 \mathrm{mg} / \mathrm{L}$. On the fourth day after hospitalization and consultation of gynecologist, patient was scheduled for laparoscopic cholecystectomy and IOUS investigation of biliary tree.

Patient was positioned in the reverse Trendelenburg position and general endotracheal anesthesia using sevoflurane, propofol and fentanyl was induced. The standard four-trocar technique was used, however, first trocar was entered by using Hasson technique and pneumoperitoneum of $12 \mathrm{mmHg}$ was maintained. During operation chronic calculous cholecystitis with swollen inflamed walls was revealed. The operation started with dissection of Callot's triangle. The cystic duct and artery were clipped and cystic artery was divided. The gallbladder was left in situ and used for retraction during the LCBDE. Using IOUS a $3 \mathrm{~mm}$ solitary cholesterol stone was detected in the distal part of the CBD (Fig.1). Transcystic LCBDE using 2.5 mm flexible choledochoscope was performed. 2.2 Fr nitinol extraction basket was used for stone removal (Fig.2). After removing the stone, clearance of the CBD was repeatedly confirmed with choledochoscopic visualization and repeated IOUS. A laparoscopic cholecystectomy was then performed using the standard method. No postoperative drains were used. Trocar insertion sites were infiltrated with $20 \mathrm{~mL}$ Chirocaine solution $(5 \mathrm{mg} / \mathrm{mL})$ for post-operative pain control. After 
the procedure patient's condition and symptoms rapidly improved, normalization of laboratory test results was achieved. Total serum bilirubin decreased to 16.04 $\mu \mathrm{mol} / \mathrm{L}$ and CRP decreased to $7.32 \mathrm{mg} / \mathrm{L}$. Postoperative course was uneventful and patient was discharged on the $5^{\text {th }}$ postoperative day. Before leaving the hospital fetal ultrasonography was performed finding no abnormalities.

Nine-month post-hospitalization period and the last two trimesters of pregnancy underwent with no postoperative complications or other acute events. Patient went into labor naturally at 39+2 and no complications occurred. Physical and cognitive development matches child's age.

\section{DISCUSSION}

Cholecystitis after appendicitis is the second most common non-obstetrical surgical emergency complicating pregnancy $(12,6,8,14)$. Gallstone formation during pregnancy can be documented up to $8 \%$ depending on the population, however, 20 $30 \%$ of de-novo formed stones dissolve spontaneously postpartum (7). Only $12 \%$ of pregnant women with gallstones will need to be hospitalized due to biliary colic and $30 \%$ of them will require surgical treatment (10).

It is proven that almost $10-18 \%$ of patients admitted with signs of symptomatic gallstone disease have concomitant stones in bile ducts $(17,5,2,15)$, although the overall incidence of choledocholithiasis in patients undergoing laparoscopic cholecystectomy is $3-14.7 \%$ $(4,16)$.

Complications associated with choledocholithiasis are relatively uncommon during pregnancy, nevertheless, they can result in significant morbidity and mortality. $(14,13)$. Certain prognostic criteria for concomitant bile duct stones have been developed, however, definition of precise management algorithm during pregnancy is still lacking.

There is more than one option for treatment of choledocholithiasis during pregnancy and one of the most common is to perform ERCP before or after gallbladder removal surgery. ERCP with its associated radiation exposure to the fetus may produce various developmental abnormalities and it is difficult to calculate a dose of fetal radiation from exposure time.

LCBDE in one-stage with laparoscopic cholecystectomy, using IOUS, can be safely performed during first and second trimester of pregnancy $(12,11)$, avoiding complications concerning mother and fetus that could accrue due to exposure of radiation. Although the laboratory values were not remarkably elevated, reported patient's clinical condition and radiologic findings showed no improvement, therefore surgical treatment was indicated. The operation was performed during the $2^{\text {nd }}$ trimester which is considered as a safe trimester for performing laparoscopic procedures (11), although according to Society of American Gastrointestinal and Endoscopic Surgeons guidelines, laparoscopic cholecystectomy can be performed safely in pregnant patients during any trimester (14).
Laparoscopic approach was considered as a better solution than open cholecystectomy because it is associated with shorter operative time, hospital stay and fewer complications (11).

In order to reduce hospital stay and risk of post-operative complications transcystic LCBDE was performed without need for choledochostomy. The indications for transcystic LCBDE include stones smaller than $9 \mathrm{~mm}$ in size, fewer than 5 in number, and cystic duct lateral entrance to CBD (18).

Overall, LCBDE with assistance of IOUS and choledochoscopy is feasible and safe procedure that can be provided during pregnancy in certain patient groups with choledocholithiasis.

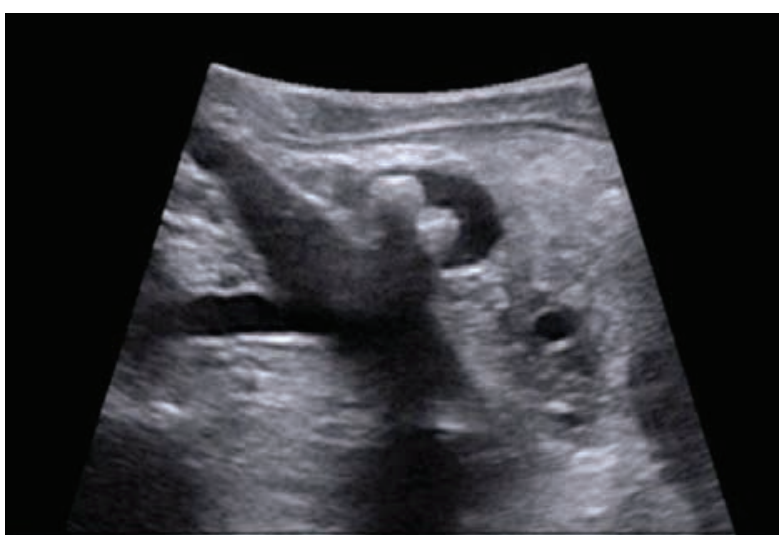

Fig. 1. Cholesterol stone in CBD

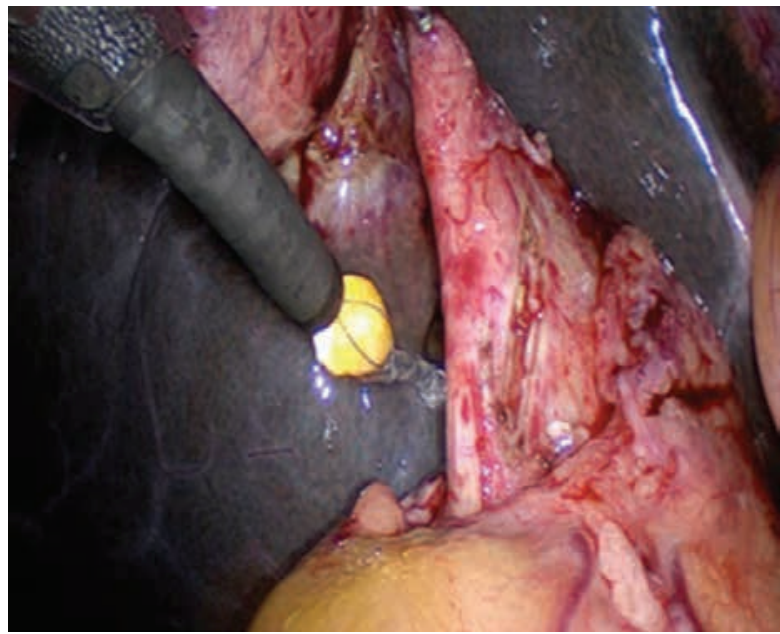

Fig. 2. Extraction of the stone with flexible choledochoscope from CBD

Conflict of interest: None 


\section{REFERENCES}

1. Anne M. Silas, Jonathan B. Kruskal, Robert A. Kane. Intraoperative Ultrasound // Radiol Clin North Am. 2001 May; 39(3):429-48

2. Attasaranya S, Fogel EL, Lehman GA. (2008) Choledocholithiasis, ascending cholangitis and gallstone pancreatitis // Med Clin North Am 92 (4): 925-960

3. Aziz O., Ashrafian H., Jones C., et al. Laparoscopic ultrasonography versus intra-operative cholangiogram for the detection of common bile duct stones during laparoscopic cholecystectomy: a meta-analysis of diagnostic accuracy // Int J Surg. 2014;12(7):712-9

4. Collins C., Maguire D., Ireland A., et al. A Prospective Study of Common Bile Duct Calculi in Patients Undergoing Laparoscopic Cholecystectomy // Ann Surg. 2004 Jan; 239(1): 28-33

5. De Palma GD. Minimally invasive treatment of cholecysto-choledocal lithiasis: The point of view of the surgical endoscopist // World J Gastrointest Surg. 2013 Jun 27; 5(6): 161-166

6. Gallstones in pregnancy. http://www.uptodate. com/contents/gallstones-in-pregnancy. Accessed 10 Jan 2016

7. Gilat T., Konikoff F. Pregnancy and the biliary tract // Can J Gastroenterol. 2000 Nov; 14 Suppl D:55D59D

8. Glanc P., Maxwell C. Acute Abdomen in Pregnancy // J Ultrasound Med 2010; 29:1457-1468

9. Guarise A., Baltieri S., Mainardi P., et al. Diagnostic accuracy of MRCP in choledocholithiasis // Radiol Med. 2005 Mar;109(3):239-51

10. Mendez-Sanchez N, Chavez-Tapia NC, Uribe M. Pregnancy and gallbladder disease // Ann Hepatol 2006; 5:227-30

11. Nasioudis D., Tsilimigras D., Economopoulos KP. Laparoscopic cholecystectomy during pregnancy: A systematic review of 590 patients // Int J Surg. 2016 Jan 28; 27:165-175
12. Parangi S., Pories S. Surgical Problems in the Pregnant Patient // In: Pregnancy in Gastrointestinal Disorders. $2^{\text {nd }}$ ed. Washington: American College of Gastroenterology; 2007; 54-57

13. Pasquale L., Caserta L., Rispo A., et al. Endoscopic management of symptomatic choledocholithiasis in pregnancy without the use of radiations // Eur Rev Med Pharmacol Sci 2007; 1 1:343-346

14. Pearl J., Price R., Richardson W., et al. Guidelines for diagnosis, treatment, and use of laparoscopy for surgical problems during pregnancy // Surg Endosc. 2011 Nov; 25(11):3479-92

15. Schreurs WH, Juttmann JR, Stuifbergen WN, Oostvogel HJ, van Vroonhoven TJ. Management of common bile duct stones: short- and longterm results with selective endoscopic retrograde cholangiography and endoscopic sphincterotomy // Surg Endosc. 2002; 16(7): 1068-1072

16. Shojaiefard A., Esmaeilzadeh M., Ghafouri A., et al. Various Techniques for the Surgical Treatment of Common Bile Duct Stones: A Meta Review // Gastroenterol Res Pract. 2009; 2009:840208. doi: 10.1155/2009/840208

17. Trondsen E, Edwin B, Reiertsen O, Faerden AE, Fagertun H, Rosseland AR. Prediction of common bile duct stones prior to cholecystectomy: a prospective validation of a discriminant analysis function // Arch Surg. 1998; 133(2): 162-166

18. Zhang W., Xu G., Huang Q., et al. Treatment of gallbladder stone with common bile duct stones in the laparoscopic era // BMC Surg. 2015; 15:7

\section{Address:}

Janis Lacis

Riga Stradins University

Dzirciema Street 16, LV-1007, Riga, Latvia

E-mail: lacisjanis@inbox.lv 